\title{
Topological Cohen-Macaulay criteria for monomial ideals
}

\author{
Ezra Miller
}

\section{Introduction}

Scattered over the past few years have been several occurrences of simplicial complexes whose topological behavior characterize the Cohen-Macaulay property for quotients of polynomial rings by arbitrary (not necessarily squarefree) monomial ideals. It is unclear whether researchers thinking about this topic have, to this point, been aware of the full spectrum of related developments. Therefore, the purpose of this survey is to gather the developments into one location, with self-contained proofs, including direct combinatorial topological connections between them.

Four families of simplicial complexes are defined in reverse chronological order: via distraction, the Čech complex, Alexander duality, and then the Koszul complex. Each comes with historical remarks and context, including forays into Stanley decompositions, standard pairs, $A$-hypergeometric systems, cellular resolutions, the Cech hull, polarization, local duality, and duality for $\mathbb{Z}^{n}$-graded resolutions.

Results or definitions appearing here for the first time include the general categorical definition of cellular complex in Definition 3.2, as well as the statements and proofs of Lemmas 3.9 and 3.10, though these are very easy. The characterization of exponent simplicial complexes in Corollary 2.9 might be considered new; certainly the consequent connection in Theorem 4.1 to the Čech simplicial complexes is new, as is the duality in Theorem 6.3 between these and the dual Cech simplicial complexes. The geometric connection between distraction and local cohomology in Theorem 4.7, and its consequences in Section 5, generalize and refine results from [BeMa08], in addition to providing commutative proofs. Finally, the connection between Cech and Koszul simplicial complexes in Lemma 7.2 and Corollary 7.8, as well as its general $\mathbb{Z}^{n}$-graded version in Theorem 7.7, appear to be new.

\section{Monomial ideals and simplicial complexes}

Throughout this article, $I$ is an ideal in the polynomial $\operatorname{ring} S=\mathbb{k}\left[x_{1}, \ldots, x_{n}\right]$. The coefficient field $\mathbb{k}$ is assumed arbitrary unless explicitly stated otherwise. Each

1991 Mathematics Subject Classification. Primary: 13F55, 13C14, 13D45, 55U10, 13-02; Secondary: 13D02, 13N10, 55U30, 13D07, 55N05, 55U15, 18E99, 18G10, 13-06.

Key words and phrases. monomial ideal, Cohen-Macaulay, simplicial complex, local cohomology, distraction, Alexander duality.

The author was partially funded by NSF CAREER Grant DMS-0449102. 
monomial in $S$ can be expressed uniquely as $\mathbf{x}^{\mathbf{a}}=x_{1}^{a_{1}} \cdots x_{n}^{a_{n}}$ for some vector $\mathbf{a}=$ $\left(a_{1}, \ldots, a_{n}\right) \in \mathbb{N}^{n}$ of $n$ nonnegative integers. More generally, Laurent monomials in $S\left[x_{1}^{-1}, \ldots, x_{n}^{-1}\right]$ can be expressed as $\mathbf{x}^{\mathbf{a}}$ for vectors $\mathbf{a} \in \mathbb{Z}^{n}$ of arbitrary integers.

For any subset $F \subseteq\{1, \ldots, n\}$ of indices, let $\mathbb{N}^{F} \subseteq \mathbb{Z}^{F}$ denote the coordinate subspace of vectors whose entries are zero outside of those indexed by $F$. This means that $\mathbb{Z}^{F}$ consists of the vectors whose support, by definition the $\operatorname{set} \operatorname{supp}(\mathbf{a})$ of indices $i$ for which $a_{i}$ is nonzero, is contained in $F$. Identify the set $F$ of indices with its characteristic vector in $\mathbb{N}^{n}$, which is the vector with support $F$ and all nonzero entries equal to 1 . Thus every squarefree monomial is $\mathbf{x}^{F}=\prod_{i \in F} x_{i}$ for some index set $F$. The inverse of such a monomial is $\mathbf{x}^{-F}$, so $S\left[\mathbf{x}^{-F}\right]=S\left[x_{i}^{-1} \mid i \in F\right]$ is a monomial localization of $S$, which we denote by $S_{F}$. In addition, let

$$
\mathfrak{m}^{F}=\left\langle\mathbf{x}_{F}\right\rangle=\left\langle x_{i} \mid i \in F\right\rangle
$$

be the ideal generated by the variables indexed by $F$. Thus $S / \mathfrak{m}^{F}=\mathbb{k}\left[\mathbf{x}_{\bar{F}}\right]$ is a polynomial ring in variables $\mathbf{x}_{\bar{F}}$ indexed by the complementary set $\bar{F}=\{1, \ldots, n\} \backslash F$.

For any ideal $I \subseteq S$, define the zero set

$$
Z(I)=\left\{\alpha \in \mathbb{k}^{n} \mid f(\alpha)=0 \text { for all } f \in I\right\} .
$$

If $I$ is generated by monomials, for example, then $Z(I)$ is a union of coordinate subspaces of $\mathbb{k}^{n}$. Conversely, if any union $Z$ of coordinate subspaces is given, then the ideal $I(Z)$ of polynomials vanishing on $Z$ is a monomial ideal. (When $\mathbb{k}$ is finite, this statement must be interpreted in the appropriate scheme-theoretic way; but there is no harm in simply assuming that $\mathbb{k}$ is algebraically closed - and hence infinite - for the duration of this article.) In fact, $I(Z)$ is a squarefree monomial ideal: its minimal monomial generators are all squarefree. On the other hand, a union $Z$ of coordinate subspaces also gives rise to a simplicial complex

$$
\Delta(Z)=\left\{F \subseteq\{1, \ldots, n\} \mid \mathbb{k}^{F} \subseteq Z\right\},
$$

meaning that $\Delta(Z)$ is a collection of subsets, called faces, that is closed under inclusion: $F \in \Delta(Z)$ and $G \subseteq F$ implies $G \in \Delta(Z)$. The assignments $Z \rightsquigarrow I(Z)$ and $Z \rightsquigarrow \Delta(Z)$ induce a bijection, called the Stanley-Reisner correspondence, between squarefree monomial ideals and simplicial complexes. Given a simplicial complex $\Delta$, the corresponding monomial ideal $I_{\Delta}$ is its Stanley-Reisner ideal (also known as its face ideal); given a squarefree monomial ideal, $\Delta_{I}$ is its Stanley-Reisner complex.

Background on squarefree monomial ideals and simplicial complexes can be found in Chapter II of Stanley's book [Sta96], or in Chapter 5 of the book by Bruns and Herzog [BrHe93], or in Chapter 1 of the book by Miller and Sturmfels [MiSt05]. The starting point for this survey is the following.

Definition 1.1. A simplicial complex $\Delta$ is Cohen-Macaulay over $\mathbb{k}$ if the StanleyReisner ring $\mathbb{k}[\Delta]$ is Cohen-Macaulay.

The Cohen-Macaulay condition on $\Delta$ is combinatorial, as discovered by Reisner.

Theorem 1.2 (Reisner's criterion, [Rei76]). A simplicial complex $\Delta$ is CohenMacaulay over $\mathbb{k}$ if and only if, for every face $F \in \Delta$, the subcomplex

$$
\operatorname{link}(F, \Delta)=\{G \in \Delta \mid G \cup F \in \Delta \text { and } G \cap F=\varnothing\},
$$

known as the link of $F$ inside $\Delta$, has reduced cohomology

$$
\widetilde{H}^{i}(\operatorname{link}(F, \Delta) ; \mathbb{k})=0 \quad \text { for } i \neq \operatorname{dim}(\Delta)-|F| \text {. }
$$


Proof. This follows from Theorem 3.1, below; alternatively, see [Sta96, Corollary II.4.2], [BrHe93, Corollary 5.3.9], or [MiSt05, Section 13.5.2].

REMARK 1.3. As combinatorial as Reisner's criterion may appear, in reality it is topological: it is equivalent to the vanishing of all but the top reduced cohomology of $\Delta$ over $\mathbb{k}$ and the top local cohomology over $\mathbb{k}$ near every point $p$ in the geometric realization $|\Delta|$; that is, the relative cohomology with coefficients in $\mathbb{k}$ of the pair $(|\Delta|,|\Delta| \backslash\{p\})[$ Sta96, Proposition II.4.3].

REMARK 1.4. Note how the field $\mathbb{k}$ enters into the definition: although the presentation of the ring $\mathbb{k}[\Delta]$ looks the same for every field $\mathbb{k}$, the Cohen-Macaulay condition can depend on the characteristic of $\mathbb{k}$ (but nothing else - all fields with the same characteristic behave just like the prime field of that characteristic). This is reflected in Reisner's criterion, where the simplicial complex is independent of $\mathbb{k}$, but its cohomology is taken with coefficients in $\mathbb{k}$.

In recent years, Reisner's topological criterion for the Cohen-Macaulay condition has been generalized to settings where the monomial ideal $I$ is no longer required to be squarefree. The essential framework is to define not one simplicial complex, but a family of them indexed by subsets of $\mathbb{Z}^{n}$, or $\mathbb{k}^{n}$ in some cases, and then connect the Cohen-Macaulayness of members in this family to the homological algebra of $I$, or to the geometry of closely related ideals. The purpose of this survey is to demonstrate direct equivalences between various families of simplicial complexes arising this way.

\section{Distracting arrangements and exponent complexes}

The first step is to produce a single family of simplicial complexes whose CohenMacaulayness reflects that of a given monomial ideal $I$, which we fix from now on.

Definition 2.1. The monomials in $S$ outside of $I$ are called standard monomials. Write $\Lambda_{I} \subseteq \mathbb{N}^{n}$ for the set of exponent vectors on the standard monomials of $I$.

LEMMA 2.2. The standard monomials form a $\mathbb{k}$-vector space basis for $S / I$.

The geometry of $\Lambda_{I}$ closely reflects the geometry of $S / I$. When $I$ is squarefree, for example, $\Lambda_{I}$ is the union of the coordinate subspaces $\mathbb{N}^{F}$ for the faces $F \in \Delta_{I}$ in the Stanley-Reisner simplicial complex, while the zero set $Z(I)$ is the union of the coordinate subspaces $\mathbb{k}^{F}$ for the faces $F \in \Delta_{I}$. In general, $\Lambda_{I}$ "looks" like the zero scheme of $I$, rather than the zero set $Z(I)$. Nonetheless, the transition from standard monomials to subspace arrangement generalizes to arbitrary monomial ideals, as long as $\mathbb{k}$ has characteristic zero (or characteristic bigger than the greatest exponent on any minimal generator of $I$ ). For simplicity, we shall work with the field $\mathbb{k}=\mathbb{C}$ of complex numbers.

Definition 2.3. The distracting arrangement $V_{I}$ of the monomial ideal $I$ is the Zariski closure $Z\left(I\left(\Lambda_{I}\right)\right) \subseteq \mathbb{C}^{n}$ of the subset $\Lambda_{I} \subseteq \mathbb{N}^{n} \subseteq \mathbb{C}^{n}$.

Taking this Zariski closure has the same feel as the squarefree case. For example, if a lattice point lies along a line containing infinitely many points in $\Lambda_{I}$, then the whole line is contained in $V_{I}$. More generally, $V_{I}$ has the following description.

LEMma 2.4. $\Lambda_{I}$ can be expressed as a finite disjoint union of translates $\mathbf{a}+\mathbb{N}^{F}$ of coordinate subspaces of $\mathbb{N}^{n}$. For any such decomposition, the distracting arrangement is the corresponding union of translates $\mathbf{a}+\mathbb{C}^{F}$ of coordinate subspaces of $\mathbb{C}^{n}$. 
The proof, and a fair bit of the rest of this survey, uses $\mathbb{Z}^{n}$-gradings; see [MiSt05, Chapter 8]. In particular, $S=\bigoplus_{\mathbf{b} \in \mathbb{Z}^{n}} S_{\mathbf{b}}$, where $S_{\mathbf{b}}$ is the 1-dimensional vector space spanned by the monomial $\mathbf{x}^{\mathbf{b}}$ if $\mathbf{b} \in \mathbb{N}^{n}$, and $S_{\mathbf{b}}=0$ if $\mathbf{b} \in \mathbb{Z}^{n} \backslash \mathbb{N}^{n}$.

Proof. Every $\mathbb{Z}^{n}$-graded $S$-module has a monomial associated prime [MiSt05, Proposition 8.11], and hence a $\mathbb{Z}^{n}$-graded submodule that is a $\mathbb{Z}^{n}$-graded translate $\mathrm{x}^{\mathrm{a}} \cdot S / \mathrm{m}^{F}$ of a quotient of $S$ modulo a monomial prime. By Noetherian induction, $S / I$ possesses a filtration by $\mathbb{Z}^{n}$-graded submodules whose associated graded pieces have this form. The first sentence is thus a consequence of Lemma 2.2, which implies that the monomials with exponent vectors in $\mathbf{a}+\mathbb{N}^{F}$ form a basis for $\mathbf{x}^{\mathbf{a}} \cdot S / \mathfrak{m}^{F}$. The second sentence follows from the first, since Zariski closure commutes with finite unions.

Unions as in Lemma 2.4 are called Stanley decompositions of $S / I$. They are relatively easy to come by, although finding ones with good properties is difficult: Stanley's conjecture [Sta82], which posits that one exists in which the minimum size of any appearing $F$ is at least the depth of $S / I$, is still open; see [HVZ08] for recent work on this topic and more background.

Locally near every point in $\mathbb{C}^{n}$, the distracting arrangement $V_{I}$ looks like a coordinate subspace arrangement. Here is a more precise statement; see later in this section for history and context.

Definition 2.5. For $\alpha \in \mathbb{C}^{n}$, let $Z_{\alpha} \subseteq V_{I}$ be the union of the components of $V_{I}$ passing through $\alpha$. Then $-\alpha+Z_{\alpha}$ is a coordinate subspace arrangement in $\mathbb{C}^{n}$, and $I\left(-\alpha+Z_{\alpha}\right)$ is the Stanley-Reisner ideal of the exponent simplicial complex $\mathrm{E} \Delta_{\alpha}$.

REMARK 2.6. Given a fixed set of monomial generators, the ideal $I \subseteq S$ can have different homological properties depending on the characteristic of $\mathbb{k}$; see Remark 1.4. The simplicial complexes $\mathrm{E} \Delta_{\alpha}$ for $\alpha \in \mathbb{C}^{n}$, on the other hand, by definition do not make reference to an arbitrary field $\mathbb{k}$. It may therefore come as a surprise that the topological invariants of $\mathrm{E} \Delta_{\alpha}$ for $\alpha \in \mathbb{Z}^{n}$ control the homological properties of $S / I$ for any field $\mathbb{k}$; see Theorems 3.8, 4.1, and 4.7, as well as Corollary 5.1. But this is analogous to the squarefree case, where the Stanley-Reisner complex is defined without reference to $\mathbb{k}$, whereas its homological invariants depend on $\mathbb{k}$; it justifies the restriction to $\mathbb{k}=\mathbb{C}$ in Definition 2.3.

Although there is no known canonical way to choose a Stanley decomposition, a measure of uniqueness can be imposed by dispensing with the disjointness hypothesis. As a matter of terminology, a cover of any set $\Lambda$ is a family of subsets whose union is $\Lambda$. One cover dominates another if every subset from the second cover is contained in a subset from the first.

Proposition 2.7. $\Lambda_{I}$ has a unique cover by translates $\mathbf{a}+\mathbb{N}^{F}$ of coordinate subspaces of $\mathbb{N}^{n}$ dominating every other such cover. Each standard pair $\mathbf{a}+\mathbb{N}^{F}$ in this standard pair decomposition of $\Lambda_{I}$ is the intersection with $\Lambda_{I}$ of an irreducible component $\mathbf{a}+\mathbb{C}^{F}$ of the distracting arrangement $V_{I}$, and $\mathbf{a}$ has support disjoint from $F$.

Proof. Every component $\mathbf{a}+\mathbb{C}^{F}$ of $V_{I}$ is the closure of a translated orthant $\mathbf{a}+\mathbb{N}^{F}$ for some $\mathbf{a} \in \mathbb{N}^{n}$, by Lemma 2.4. Observe that $\left(\mathbf{a}+\mathbb{C}^{F}\right) \cap \mathbb{N}^{n}=\mathbf{a}_{F}+\mathbb{N}^{F}$, where $\mathbf{a}_{F}$ is obtained from a by setting to 0 the coordinates of $\mathbf{a}$ indexed by $\bar{F}$. Once $\Lambda_{I}$ contains $\mathbf{a}+\mathbb{N}^{F}$, it contains $\mathbf{a}_{F}+\mathbb{N}^{F}$, because the standard monomials are closed under divisibility: every divisor of every standard monomial is standard. 
Standard pair decompositions were introduced by Sturmfels, Trung, and Vogel [STV95, Section 3], for the reason that the number of times $F$ appears equals the multiplicity of the prime ideal $\mathfrak{m}^{\bar{F}}$ in the primary decomposition of $I$. Later, standard pairs became the device through which the exponent simplicial complexes $\mathrm{E} \Delta_{\alpha}$ were connected to the Cohen-Macaulay property.

REMARK 2.8. The earliest context in which exponent simplicial complexes seem to have appeared explicitly, in the form of Definition 2.5, was in the noncommutative algebra of multivariate hypergeometric systems [SST00]. The question of characterizing the Cohen-Macaulay condition had arisen naturally. The combinatorial commutative context there was more general than the one here (it was for affine semigroup rings), but it led back to distractions, nonetheless, as follows.

Associated to an affine semigroup is a family, parametrized by $\mathbb{C}^{d}$, of systems of linear partial differential equations with polynomial coefficients [GGZ87, GKZ89]. Based on observations by Gelfand, Kapranov, and Zelevinsky, along with subsequent work by Adolphson [Ado94], Sturmfels conjectured that the systems in this family all have the same number of linearly independent solutions precisely when the corresponding affine semigroup ring over the complex numbers is Cohen-Macaulay.

This conjecture was proved by Matusevich, Miller, and Walther [MMW05] by introducing functorial noncommutative Koszul-like homological methods for $\mathbb{Z}^{d_{-}}$ graded modules over semigroup rings: the rank of the solution space jumps whenever the parameter in $\mathbb{C}^{d}$ lies in a certain subspace arrangement. The arrangement is a quasidegree set as in Corollary 5.10, coming from local cohomology just as distractions come from monomial quotients. In particular, the arrangement is nonemptyso there exists at least one rank jumping parameter in $\mathbb{C}^{d}$-precisely when there is nonzero local cohomology to indicate the failure of Cohen-Macaulayness.

Berkesch and Matusevich [BeMa08] used an extension [DMM08] of these homological methods to more general multigraded modules over polynomial rings to see what the Cohen-Macaulay characterization by rank jumps says about quotients by monomial ideals. Their conclusions were based on the fact that ranks of systems of differential equations arising from monomial ideals are controlled by the geometry of their distractions, from which Berkesch and Matusevich defined the exponent simplicial complexes using standard pairs. Sections 4 and 5 contain details about the results in [BeMa08]; for now, the reader is encouraged to consult that article for colorful illustrations of distracting arrangements and exponent simplicial complexes.

Proposition 2.7 results in an alternate characterization of exponent complexes that will be useful in the comparisons with other simplicial complexes to come.

Corollary 2.9. A subset $F \subseteq\{1, \ldots, n\}$ is a maximal face (that is, a facet) of the exponent complex $\mathrm{E} \Delta_{\alpha}$ of $I$ if and only if the set $\left(\alpha+\mathbb{C}^{F}\right) \cap \Lambda_{I}$ of lattice points is dense in $\alpha+\mathbb{C}^{F}$ and $F$ is maximal with this property.

Proof. $F$ is a facet of $\Delta_{\alpha}$ if and only if $\alpha+\mathbb{C}^{F}$ is an irreducible component of the distracting arrangement $V_{I}$, by Definition 2.5. If $\alpha+\mathbb{C}^{F}$ is an irreducible component of $V_{I}$, then $\left(\alpha+\mathbb{C}^{F}\right) \cap \Lambda_{I}$ is a dense subset of it by Proposition 2.7, and $F$ is maximal with this property because otherwise $\alpha+\mathbb{C}^{F}$ would be strictly contained in a component $\alpha+\mathbb{C}^{F^{\prime}}$ for some other face $F^{\prime}$. On the other hand, if $\left(\alpha+\mathbb{C}^{F}\right) \cap \Lambda_{I}$ is dense in $\alpha+\mathbb{C}^{F}$, then $\alpha+\mathbb{C}^{F} \subseteq V_{I}$ by Definition 2.3 ; maximality of $F$ guarantees that $\alpha+\mathbb{C}^{F}$ is a component, given that each component of $V_{I}$ is parallel to a coordinate subspace of $\mathbb{C}^{n}$, which follows from Proposition 2.7, or even Lemma 2.4. 


\section{3. Čech simplicial complexes}

One of the many characterizations of the Cohen-Macaulay condition [MiSt05, Theorem 13.37] for a module $M$ is by vanishing of all but the top-that is, cohomological degree $\operatorname{dim}(M)$-local cohomology $H_{\mathfrak{m}}^{i}(M)$ supported on the maximal ideal $\mathfrak{m}$ of $S$. In particular, Reisner's criterion (Theorem 1.2) is a consequence of the following famous combinatorial formula for local cohomology.

TheOREM 3.1 (Hochster's formula). The $\mathbb{Z}^{n}$-graded Hilbert series of the local cohomology with maximal support of a Stanley-Reisner ring satisfies

$$
H\left(H_{\mathfrak{m}}^{i}\left(S / I_{\Delta}\right) ; \mathbf{x}\right)=\sum_{F \in \Delta} \operatorname{dim}_{\mathbb{k}} \widetilde{H}^{i-|F|-1}(\operatorname{link}(F, \Delta) ; \mathbb{k}) \prod_{j \in F} \frac{x_{j}^{-1}}{1-x_{j}^{-1}} .
$$

Proof. This can be deduced from Theorem 3.8, below; alternatively, see [Sta96, Theorem II.4.1], [BrHe93, Theorem 5.3.8], or [MiSt05, Theorem 13.13].

The proof of this statement is remarkably straightforward: the Cech complex of $S / I_{\Delta}$ is a complex of $\mathbb{Z}^{n}$-graded $S$-modules, whose graded piece in degree a is the cochain complex of the appropriate link in $\Delta$, with a homological shift. This argument is a quintessential example of the "cellular" technique that pervades a significant portion of combinatorial commutative algebra in recent years. Quite generally, this technique begins with a cell complex $X$, which could be a CW complex, a simplicial complex, a polyhedral complex, or any other desired type of cell complex. This topological cell complex $X$ has an algebraic chain complex

$$
\text { C.X }: \quad 0 \leftarrow \bigoplus_{\text {vertices } v \in X} \mathbb{Z}_{v} \leftarrow \bigoplus_{\text {edges } e \in X} \mathbb{Z}_{e} \leftarrow \cdots \leftarrow \bigoplus_{i \text {-faces } \sigma \in X} \mathbb{Z}_{\sigma} \leftarrow \cdots
$$

over the integers, where $\mathbb{Z}_{\tau} \leftarrow \mathbb{Z}_{\sigma}$ is multiplication by some integer coeff $(\sigma, \tau)$.

Definition 3.2. Fix a cell complex $X$, an abelian category, an object $J_{\sigma}$ for each face $\sigma \in X$, and a natural map $J_{\tau} \leftarrow J_{\sigma}$ for each inclusion $\tau \subseteq \sigma$ of faces. The complex

$$
J_{\bullet}: \quad 0 \leftarrow \bigoplus_{\text {vertices } v \in X} J_{v} \leftarrow \bigoplus_{\text {edges } e \in X} J_{e} \leftarrow \cdots \leftarrow \bigoplus_{i \text {-faces } \sigma \in X} J_{\sigma} \leftarrow \cdots
$$

is cellular and supported on $X$ if the component $J_{\tau} \leftarrow J_{\sigma}$ is coeff $(\sigma, \tau)$ times the given natural map. A similar construction yields cocellular complexes, starting from the algebraic cochain complex of $X$ and the natural maps are $J_{\tau} \rightarrow J_{\sigma}$ for $\tau \subseteq \sigma$.

REMARK 3.3. There are more variations than those mentioned in Definition 3.2. For example, we could start from the reduced (or augmented) algebraic chain or cochain complex of a simplicial complex, which includes another (co)homological degree for the empty face of $X$. We could also start from the algebraic relative (co)chain complex of a pair of cell complexes. Generally speaking, any complex in an abelian category constructed from underlying topological data on a cell complex, or pair of cell complexes, is referred to as a cellular complex of objects in that category.

EXAMPLE 3.4. The prototypical example is the algebraic cochain complex arising from Čech cohomology theory in topology. In this case, the cell complex $X$ is a simplex, and $J_{\sigma}$ is a space of functions on an intersection $\bigcap_{j \in \sigma} U_{j}$ of open sets in a given cover $\left\{U_{1}, \ldots, U_{n}\right\}$ of a fixed topological space (usually unrelated to $X$ ).

In its $\mathbb{Z}^{n}$-graded avatar, the Cech complex $\check{\mathcal{C}}^{\bullet}(\mathbf{x})$ is a cocellular complex of monomial localizations of $S$ supported on a simplex $X$. More precisely, $X$ is the 
simplex on $\{1, \ldots, n\}$, and $J_{F}=S_{F}$ is the monomial localization $S\left[\mathbf{x}^{-F}\right]$, the natural map $S_{F} \rightarrow S_{F^{\prime}}$ being the localization homomorphism for $F \subseteq F^{\prime}$.

REMARK 3.5. Other cellular complexes have played roles in commutative algebra for well over half a century, perhaps the first being the Koszul complex [Kos50], which was originally introduced for the purpose of Lie algebra cohomology theory. The initial use of cellular complexes in commutative algebra that is identifiably combinatorial came with the Taylor complex [Tay60], which provides a free resolution of any monomial ideal in a polynomial ring. The application of Čech complexes to local cohomology of monomial ideals was carried out in the 1970's. The earliest cellular complexes supported on polyhedra that are not simplicial complexes arrived in the work of Ishida on dualizing complexes and local cohomology with maximal support over affine semigroup rings [Ish80, Ish87]; in addition, Ishida's objects $J_{\sigma}$ were modules over rings other than polynomial rings. The notion of cellular resolution in combinatorial commutative algebra was codified formally for the first time in the work of Bayer and Sturmfels, also with Peeva [BaSt98, BPS98], in the context of lattice ideals and generic monomial ideals (the more inclusive definition of generic monomial ideals in current use [MSY00] was introduced later).

More general objects $J_{\sigma}$, including graded injectives and quotients by irreducible monomial ideals in polynomial and affine semigroup rings, began to appear in [Mil00] and [Mil02b], where topological duality for the support complexes was observed to reflect algebraic (Matlis or local) duality algebraically. All of the cellular resolutions to that point had been supported on special classes of cell complexes, notably simplicial and polyhedral complexes. With the work of Batzies and Welker [BaWe02], more flexible support complexes $X$ began appearing, allowing the application of homotopic and Morse-theoretic cancellative approaches, which don't preserve polyhedrality. Cellular methods generally bring topological or combinatorial techniques to bear on algebraic problems, but Fløystad, with his enriched cohomology, has begun to realize that commutative algebra can provide additional homological insight into the cell complex $X$, rather than vice versa [Flø06]. Most recently, cellular techniques in combinatorial commutative algebra have given way to non-combinatorial applications where the objects $J_{\sigma}$ do not belong to commutative algebra at all: in [JoMi08], where Definition 3.2 was mentioned in the Introduction without details, they are sheaves from scheme-theoretic algebraic geometry, or perhaps from complex analytic geometry.

These examples are idiosyncratic choices of landmarks; there have been many others between, demonstrating the strengths and limitations of cellular resolutions.

Generalizing Theorem 3.1 to the local cohomology of $S / I$ for an arbitrary monomial ideal $I$ involves simplicial complexes that can be characterized as follows.

Definition 3.6. Given $\mathbf{a} \in \mathbb{Z}^{n}$, write $\mathbf{a}=\mathbf{a}_{+}-\mathbf{a}_{-}$as a difference of vectors in $\mathbb{N}^{n}$. The Čech simplicial complex of $I$ in degree $\mathbf{a}$ is the set

$$
\check{\mathrm{C}} \Delta_{\mathbf{a}}=\left\{F-\operatorname{supp}\left(\mathbf{a}_{-}\right) \mid\left(S_{F} / I S_{F}\right)_{\mathbf{a}}=\mathbb{k}\right\}
$$

in bijection with those $F$ for which the localization $S / I \otimes_{S} S_{F}$ is nonzero in degree a.

LEMMA 3.7. $\check{C} \Delta_{\mathbf{a}}$ is a simplicial complex.

Proof. For $F \subseteq F^{\prime}$, the homomorphism from $S_{F} / I S_{F}$ to its image in $S_{F^{\prime}} / I S_{F^{\prime}}$ is the quotient homomorphism of $S_{F} / I S_{F}$ modulo the kernel of the localization 
that inverts $\mathbf{x}^{F^{\prime}-F}$. Therefore, once $S_{F}$ is nonzero in degree $\mathbf{a}$, the homomorphism $\left(S_{F} / I S_{F}\right)_{\mathbf{a}} \rightarrow\left(S_{F^{\prime}} / I S_{F^{\prime}}\right)_{\mathbf{a}}$ is a quotient map. The final observation to make is that $S_{F}$ is nonzero in degree a precisely when $F$ contains $\operatorname{supp}\left(\mathbf{a}_{-}\right)$.

TheOrem 3.8. The Čech simplicial complexes $\check{C} \Delta_{\mathbf{a}}$ of I compute local cohomology:

$$
H_{\mathfrak{m}}^{i}(S / I)_{\mathbf{a}}=\widetilde{H}^{i-\left|\operatorname{supp}\left(\mathbf{a}_{-}\right)\right|-1}\left(\check{C}_{\mathbf{a}_{\mathbf{a}}} ; \mathbb{k}\right) .
$$

Proof. The local cohomology $H_{\mathfrak{m}}^{i}(S / I)_{\mathbf{a}}$ is the $\mathbb{Z}^{n}$-degree a piece of the Čech complex $S / I \otimes_{S} \check{\mathcal{C}} \bullet(\mathbf{x})$. Viewing the Cech complex as being cocellular as in Example 3.4, and using that $S / I \otimes_{S} S_{F}=S_{F} / I S_{F}$, the degree a piece of $S / I \otimes_{S} \check{\mathcal{C}}^{\bullet}(\mathbf{x})$ is the reduced cochain complex of $\check{\mathrm{C}} \Delta_{\mathbf{a}}$ by Definition 3.6, with the faces $F-\operatorname{supp}\left(\mathbf{a}_{-}\right)$of dimension $i-\left|\operatorname{supp}\left(\mathbf{a}_{-}\right)\right|-1$ contributing to cohomological degree $i=|F|$.

Theorem 3.8 was recorded by Takayama [Tak05, Theorem 1], as the first step in studying locally Cohen-Macaulay monomial schemes - that is, whose local cohomology modules $H_{\mathfrak{m}}^{i}(S / I)$ have finite length for $i<\operatorname{dim}(S / I)$. Takayama also proved, using the simplicial formula, that $H_{\mathfrak{m}}^{i}(S / I)$ is nonzero only in degrees $\mathbf{a} \in \mathbb{Z}^{n}$ satisfying $\mathbf{a} \preceq \mathbf{a}_{I}-\mathbb{1}$, where $\mathbf{a}_{I}$ is the exponent on the least common multiple of the minimal generators, $\mathbb{1}=(1, \ldots, 1)$, and the relation $\preceq$ is coordinatewise dominance. This obsesrvation admits a simple proof in the general context of arbitrary finitely generated $\mathbb{Z}^{n}$-graded modules by using $\mathbb{Z}^{n}$-graded injective resolutions (see [MiSt05, Chapter 11] for an introduction). The general statement is as follows.

LEMma 3.9. If the Betti numbers $\beta_{i, \mathbf{b}}(M)$ of a finitely generated $\mathbb{Z}^{n}$-graded module $M$ lie in degrees $\mathbf{b} \preceq \mathbf{a}$, then $H_{\mathfrak{m}}^{i}(M)$ can only be nonzero in $\mathbb{Z}^{n}$-degrees $\preceq \mathbf{a}-\mathbb{1}$.

Proof. The proof consists of two observations:

(i) the Betti number $\beta_{i, \mathbf{b}}(M)$ equals the Bass number $\mu_{n-i, \mathbf{b}-\mathbb{1}}(M)$ at the maximal ideal, as can be seen by using a Koszul complex to compute both; and

(ii) the $\mathbb{Z}^{n}$-graded injective hull of $\mathbb{k}$ is nonzero only in degrees from $-\mathbb{N}^{n}$.

In fact, (i) implies more, when $M$ is graded by $\mathbb{N}^{n}$, and not just $\mathbb{Z}^{n}$.

Lemma 3.10. If $M$ is $\mathbb{N}^{n}$-graded and $H_{\mathfrak{m}}^{i}(M)$ is finitely generated (i.e., has finite length), then the $\mathbb{Z}^{n}$-graded translate $H_{\mathfrak{m}}^{i}(M)(-\mathbb{1})$ up by $(1, \ldots, 1)$ is also $\mathbb{N}^{n}$-graded.

Proof. The Čech hull [Mil00, Definition 2.7] (or see [MiSt05, Definition 13.32]) of any $\mathbb{Z}^{n}$-graded module $N$ is the $\mathbb{Z}^{n}$-graded module $\check{\mathcal{C}} N$ whose degree $\mathbf{b}$ piece is $(\check{\mathcal{C}} N)_{\mathbf{b}}=N_{\mathbf{b}_{+}}$for $\mathbf{b} \in \mathbb{Z}^{n}$. The Čech hull is an exact functor on $\mathbb{Z}^{n}$-graded modules because this is measured degree-by-degree. It is easy to show that the Cech hull of any indecomposable $\mathbb{Z}^{n}$-graded injective module $E$ is either zero or $E$, the latter precisely when the $\mathbb{Z}^{n}$-graded degree piece $E_{\mathbf{0}}$ is nonzero [Mil00, Lemma 4.25]. It follows immediately from (i) that the minimal injective resolution of $M(-\mathbb{1})$ - and hence all of the local cohomology of $M(-\mathbb{1})$-is fixed by the Cech hull. The previous sentence is what the statement of the lemma should really say, since any finitely generated module fixed by the Cech hull must vanish in all degrees $\notin \mathbb{1}+\mathbb{N}^{n}$.

REMARK 3.11. Rahimi generalized Theorem 3.8 to a combinatorial formula for the local cohomology of $S / I$ supported at an arbitrary prime monomial ideal [Rah07]. It remains open to find a combinatorial formula for the local cohomology of $S / I$ supported at an arbitrary (may as well be squarefree) monomial ideal. 


\section{Local cohomology vs. distraction}

Thus far we have seen two families of simplicial complexes: the exponent (Definition 2.5) and Čech (Definition 3.6) complexes. They were introduced in radically different contexts, the former being the geometry of distractions, and the latter being the graded algebra of local cohomology; but they are very closely related.

TheOREM 4.1. The exponent simplicial complex $\mathrm{E} \Delta_{\mathbf{a}}$ of I in degree $\mathbf{a} \in \mathbb{Z}^{n}$ is a cone with apex $\operatorname{supp}\left(\mathbf{a}_{-}\right)$, and the $\check{C}$ ech simplicial complex $\check{C} \Delta_{\mathbf{a}}$ is the link of the apex:

$$
\check{C} \Delta_{\mathbf{a}}=\operatorname{link}\left(\operatorname{supp}\left(\mathbf{a}_{-}\right), \mathrm{E} \Delta_{\mathbf{a}}\right) .
$$

More generally, for $\alpha \in \mathbb{C}^{n}$, define $\alpha^{\prime} \in \mathbb{Z}^{n}$ by setting to -1 all coordinates of $\alpha$ that lie outside of $\mathbb{N}$. Then $\mathrm{E} \Delta_{\alpha}$ is a cone with apex $\alpha_{-}^{\prime}$ and $\breve{C} \Delta_{\alpha^{\prime}}=\operatorname{link}\left(\alpha_{-}^{\prime}, \mathrm{E} \Delta_{\alpha}\right)$.

Proof. First observe that $\check{\mathrm{C}} \Delta_{\mathbf{a}^{\prime}}=\check{\mathrm{C}} \Delta_{\mathbf{a}}$ for $\mathbf{a} \in \mathbb{Z}^{n}$, since the variables $x_{i}$ for $i \in \operatorname{supp}\left(\mathbf{a}_{-}\right)=\mathbf{a}_{-}^{\prime}$ are units on $S_{F} / I S_{F}$ whenever $F \supseteq \operatorname{supp}\left(\mathbf{a}_{-}\right)$. Hence the second sentence is indeed more general than the first.

Lemma 4.2. $\mathrm{E} \Delta_{\alpha^{\prime}}=\mathrm{E} \Delta_{\alpha}$ for all $\alpha \in \mathbb{C}^{n}$. More generally, if $\beta$ is obtained from $\alpha \in \mathbb{C}^{n}$ by changing some nonnegative integer entries of $\alpha$ to lie outside of $\mathbb{N}$, then $\mathrm{E} \Delta_{\beta}=\operatorname{star}\left(\operatorname{supp}(\alpha-\beta), \mathrm{E} \Delta_{\alpha}\right)$, where for any simplicial complex $\Delta$ and face $F$,

$$
\operatorname{star}(F, \Delta)=\left\{F^{\prime} \in \Delta \mid F \cup F^{\prime} \in \Delta\right\} .
$$

Proof. The first sentence follows from the second by expressing both as stars in $\mathrm{E} \Delta_{\alpha_{0}}$, where $\alpha_{0}$ is obtained from $\alpha$ by setting to 0 all entries of $\alpha$ lying outside of $\mathbb{N}$.

For $F$ to be a facet of $\mathrm{E} \Delta_{\alpha}$, it must by Corollary 2.9 contain the set of indices $i$ for which $\alpha_{i}$ lies outside of $\mathbb{N}$, or else the affine subspace $\alpha+\mathbb{C}^{F}$ fails to intersect $\mathbb{N}^{n}$, let alone being the closure of a set of lattice points therein. Therefore the facets of $\mathrm{E} \Delta_{\beta}$ are precisely the facets of $\mathrm{E} \Delta_{\alpha}$ that contain $\operatorname{supp}(\alpha-\beta)$.

The lemma reduces Theorem 4.1 to the claim that $\check{\mathrm{C}} \Delta_{\mathbf{a}}=\operatorname{link}\left(\mathbf{a}_{-}, \mathrm{E} \Delta_{\mathbf{a}}\right)$ when $\mathbf{a}^{\prime}=\mathbf{a}$. For this, it is enough by Corollary 2.9 to show, when $\mathbf{a}_{-} \subseteq F$, that $\left(S_{F} / I S_{F}\right)_{\mathbf{a}}=\mathbb{k}$ if and only if $\left(\mathbf{a}+\mathbb{C}^{F}\right) \cap \Lambda_{I}$ is dense in $\mathbf{a}+\mathbb{C}^{F} ;$ but both of these, given $\mathbf{a}_{-} \subseteq F$, are equivalent to $\mathbf{x}^{\mathbf{a}_{+}} \mathbb{C}\left[\mathbf{x}_{F}\right]$ being a subquotient of $S / I$.

Remark 4.3. Berkesch and Matusevich observed (cf. Remark 2.8) that the cohomology of the exponent simplicial complexes of $I$ controls the Cohen-Macaulay property. Their precise statement along these lines [BeMa08, Theorem 3.12] is the final claim in Corollary 5.1, below. However, they did not explicitly mention that the Cohen-Macaulay property for all exponent simplicial complexes is equivalent to the Cohen-Macaulay property for the distracting arrangement $V_{I}$, because their definition of $\mathrm{E} \Delta_{\alpha}$ was combinatorial, in contrast to the equivalent geometric definition in Section 2 here. Nevertheless, the next main result here, Theorem 4.7, which provides a precise geometric criterion for the failure of the Cohen-Macaulay condition at a point of $V_{I}$, is inspired by a similar-but weaker-result of Berkesch and Matusevich; see Corollary 5.10.

Definition 4.4. Given a $\mathbb{Z}^{n}$-graded module $N$, its true degree set is the set

$$
\operatorname{tdeg}(M)=\left\{\mathbf{a} \in \mathbb{Z}^{n} \mid N_{\mathbf{a}} \neq 0\right\}
$$

of $\mathbb{Z}^{n}$-graded degrees where $N$ is nonzero. The quasidegree set $\operatorname{qdeg}(N)$ is the Zariski closure of $\operatorname{tdeg}(N)$ inside of $\mathbb{C}^{n}$. Let $\operatorname{qdeg}_{\mathbb{R}}(N)=\operatorname{qdeg}(N) \cap \mathbb{R}^{n}$ be the real quasidegree set of $N$. 
Example 4.5. The true degree set of $S / I$ is $\operatorname{tdeg}(S / I)=\Lambda_{I}$ from Definition 2.1. The quasidegree set of $S / I$ is the distracting arrangement $V_{I}$ from Definition 2.3.

REMARK 4.6. Quasidegree sets and distracting arrangements in $\mathbb{C}^{n}$ are varieties over $\mathbb{C}$, so it makes sense to say they are Cohen-Macaulay. Their intersections with $\mathbb{R}^{n}$ are varieties over $\mathbb{R}$, but are also topological spaces. Using Remark 1.3, it therefore makes sense to say that quasidegree sets or distracting arrangements over $\mathbb{R}$ are Cohen-Macaulay over a field that might not be $\mathbb{C}$. That said, the following result is true - with the same proof - if $\mathbb{R}$ and $\mathbb{k}$ are replaced by $\mathbb{C}$ throughout.

ThEOREM 4.7. If $\operatorname{dim}(S / I)=d$, the real distracting arrangement $V_{I}(\mathbb{R})=V_{I} \cap \mathbb{R}^{n}$ is not Cohen-Macaulay over $\mathbb{k}$ near $\alpha \in \mathbb{R}^{n} \Leftrightarrow \alpha \in \operatorname{qdeg}_{\mathbb{R}}\left(H_{\mathfrak{m}}^{i}(S / I)\right)$ for some $i<d$.

Proof. The arrangement $V_{I}(\mathbb{R})$ fails to be Cohen-Macaulay over $\mathbb{k}$ near $\alpha \in \mathbb{R}^{n}$ if and only if $\mathrm{E} \Delta_{\alpha}$ fails to be Cohen-Macaulay over $\mathbb{k}$. Noting that $\operatorname{link}\left(F, \mathrm{E} \Delta_{\alpha}\right)$ remains a cone unless $F$ contains $\alpha_{-}^{\prime}$ (Theorem 4.1), this occurs precisely when there exist a face $F \in \mathrm{E} \Delta_{\alpha}$ with $F \supseteq \alpha_{-}^{\prime}$ and a cohomological degree $i<d$ such that

$$
\widetilde{H}^{i-|F|-1}\left(\operatorname{link}\left(F, \mathrm{E} \Delta_{\alpha}\right) ; \mathbb{k}\right) \neq 0
$$

by Reisner's criterion (Theorem 1.2). On the other hand, if $\mathbf{a}=\alpha_{F}$ is the result of setting to -1 all coordinates of $\alpha$ indexed by $F$, then $\operatorname{link}\left(F, \mathrm{E} \Delta_{\alpha}\right)=\operatorname{link}\left(F, \mathrm{E} \Delta_{\mathbf{a}}\right)$ by Lemma 4.2. But $\operatorname{link}\left(F, \mathrm{E} \Delta_{\mathbf{a}}\right)=\check{\mathrm{C}} \Delta_{\mathbf{a}}$ by Theorem 4.1. Furthermore, $\check{\mathrm{C}} \Delta_{\mathbf{a}}=$ $\check{\mathrm{C}} \Delta_{\mathbf{b}}$ for all $\mathbf{b} \in \mathbf{a}-\mathbb{N}^{F}$ by the first sentence of the proof of Theorem 4.1. Stringing these statements together, and applying Theorem 3.8 , we find that $V_{I}(\mathbb{R})$ fails to be Cohen-Macaulay over $\mathbb{k}$ at $\alpha$ if and only if

(*) there exist $F \supseteq \alpha_{-}^{\prime}$ and $i<d$ with $\mathbf{b} \in \operatorname{tdeg}\left(H_{\mathfrak{m}}^{i}(S / I)\right)$ for all $\mathbf{b} \in \alpha_{F}-\mathbb{N}^{F}$.

This condition certainly implies that $\alpha \in \operatorname{qdeg}_{\mathbb{R}} H_{\mathfrak{m}}^{i}(S / I)$.

For the converse, assume $i<d$ with $\alpha \in \operatorname{qdeg}_{\mathbb{R}}\left(H_{\mathfrak{m}}^{i}(S / I)\right)$. Then $\alpha+\mathbb{R}^{F}$ contains a dense subset of points in $\operatorname{tdeg}\left(H_{\mathfrak{m}}^{i}(S / I)\right)$ for some $F$ and $i<d$. This set $F$ is forced to contain the set of indices where $\alpha$ is not an integer, or else $\alpha+\mathbb{R}^{F}$ wouldn't contain any lattice points at all. Replacing $\alpha$ by some point in $\alpha+\mathbb{R}^{F}$ with $\alpha_{i}=-1$ for $i \in F$ (this changes neither $\alpha_{F}$ nor $\alpha^{\prime}$ ), we may as well assume $\alpha \in \mathbb{Z}^{n}$. Lemma 3.9 implies that $\operatorname{tdeg}\left(H_{\mathfrak{m}}^{i}(S / I)\right) \cap\left(\alpha-\mathbb{N}^{F}\right)$ contains a lattice point $\mathbf{b}$, since the Zariski closure of the complementary set $\operatorname{tdeg}\left(H_{\mathfrak{m}}^{i}(S / I)\right) \cap\left(\alpha-\left(\mathbb{Z}^{F} \backslash \mathbb{N}^{F}\right)\right)$ of lattice points in $\alpha+\mathbb{Z}^{F}$ has dimension less than $|F|$. Theorems 3.8 and $4.1 \mathrm{imply}$ that $\widetilde{H}^{i-\left|\mathbf{b}_{-}^{\prime}\right|-1}\left(\operatorname{link}\left(\mathbf{b}_{-}^{\prime}, \mathrm{E} \Delta_{\mathbf{b}}\right) ; \mathbb{k}\right) \neq 0$. Given this nonvanishing, we might as well replace $F$ with $\mathbf{b}_{-}^{\prime}$, which contains $\alpha_{-}^{\prime}$ by construction, proving $(*)$.

REMARK 4.8. The analysis in Theorem 4.7 and its proof comes down to the fact that if some exponent simplicial complex $\mathrm{E} \Delta_{\alpha}$ fails to be Cohen-Macaulay, then some link in $\mathrm{E} \Delta_{\alpha}$ has nonzero reduced cohomology in cohomological degree less than its dimension. But the Čech simplicial complex $\check{\mathrm{C}} \Delta_{\alpha}$, which is indeed a link in $\mathrm{E} \Delta_{\alpha}$, might not itself have nonzero non-top reduced cohomology. Thus, even if you're standing at a lattice point $\alpha$ whose simplicial complex fails Cohen-Macaulayness, you might not get nonvanishing local cohomology in that particular degree; instead, you might have travel along a component of $q \operatorname{deg}\left(H_{\mathfrak{m}}^{i}(S / I)\right)$, thereby taking a link, to get to a lattice point whose Čech simplicial complex does indeed have nonzero non-top local cohomology. That's the point, really: the difference between the distracting arrangement $V_{I}$ and $q \operatorname{deg}\left(H_{\mathfrak{m}}^{i}(S / I)\right)$ is the difference between possessing a face whose link witnesses failure of Cohen-Macaulayness and actually choosing one. 


\section{Geometric Cohen-Macaulay criteria}

The equivalence between exponent and Čech simplicial complexes in Theorem 4.1, and the geometric relation between the distracting arrangement and the degrees of nonvanishing local cohomology in Theorem 4.7, give rise to a number of Cohen-Macaulay criteria. Here is the first, already discussed in Remark 4.3.

Corollary 5.1. $S / I$ is Cohen-Macaulay $\Leftrightarrow$ the real distracting arrangement $V_{I}(\mathbb{R})$ is Cohen-Macaulay over $\mathbb{k}$. In fact, $S / I$ is Cohen-Macaulay of dimension $d \Leftrightarrow$ the exponent complexes $\mathrm{E} \Delta_{\alpha}$ for $\alpha \in \mathbb{C}^{n}$ are Cohen-Macaulay over $\mathbb{k}$ of dimension $d-1$.

Proof. By the well-known local cohomology criterion for Cohen-Macaulayness (see [MiSt05, Theorem 13.37.9], for example), Theorem 4.7, and Definition 2.5,

$S / I$ is Cohen-Macaulay $\Leftrightarrow H_{\mathfrak{m}}^{i}(S / I)=0$ for all $i<d$

$\Leftrightarrow \operatorname{qdeg}_{\mathbb{R}}\left(H_{\mathfrak{m}}^{i}(S / I)\right)$ is empty for all $i<d$

$\Leftrightarrow V_{I}(\mathbb{R})$ is Cohen-Macaulay over $\mathbb{k}$ at every point $\alpha \in \mathbb{R}^{n}$

$\Leftrightarrow \mathrm{E} \Delta_{\alpha}$ is Cohen-Macaulay over $\mathbb{k}$ at every point $\alpha \in \mathbb{R}^{n}$.

The result now follows from Lemma 4.2, which implies that $\left\{\mathrm{E} \Delta_{\alpha} \mid \alpha \in \mathbb{C}^{n}\right\}=$ $\left\{\mathrm{E} \Delta_{\alpha} \mid \alpha \in \mathbb{R}^{n}\right\}=\left\{\mathrm{E} \Delta_{\mathbf{a}} \mid \mathbf{a} \in \mathbb{Z}^{n}\right\}$; that is, no new exponent simplicial complexes arise by considering $\alpha \in \mathbb{C}^{n}$ instead of $\alpha \in \mathbb{R}^{n}$.

This connection between the geometry of distracting arrangements and homological properties of monomial ideals can also be seen to arise from elementary algebraic manipulation of monomials.

Definition 5.2. For a variable $t$ and $a \in \mathbb{N}$, the distraction of the pure power $t^{a}$ is

$$
\widetilde{t^{a}}=t(t-1)(t-2) \cdots(t-a+1),
$$

a polynomial of degree $a$; the distraction of $1=t^{0}$ is defined to equal 1 . For $\mathbf{a} \in \mathbb{N}^{n}$, the distraction of the monomial $\mathbf{x}^{\mathbf{a}} \in S$ is the product

$$
\widetilde{\mathrm{x}^{\mathbf{a}}}=\prod_{i=1}^{n} \widetilde{x_{i}^{a_{i}}}
$$

of the distractions of its pure-power factors $x_{i}^{a_{i}}$. For any monomial ideal $I \subseteq S$, the distraction of $I$ is the ideal $\widetilde{I}$ generated by the distractions of the monomials in $I$.

Example 5.3. The distraction of $I=\left\langle x^{3} y z^{2}, y^{2} z^{4}\right\rangle$ is

$$
\widetilde{I}=\langle x(x-1)(x-2) y z(z-1), y(y-1) z(z-1)(z-2)(z-3)\rangle .
$$

LEMma 5.4. $\widetilde{I}$ is generated by the distractions of the minimal generators of $I$.

The definitions allow for an algebraic version of Corollary 5.1.

Corollary 5.5. If $\mathbb{k}=\mathbb{C}$, then $S / I$ is Cohen-Macaulay $\Leftrightarrow$ the distraction $S / \tilde{I}$ is.

Proof. $S / \tilde{I}$ is the complex coordinate ring of the distracting arrangement $V_{I}$.

Why should the algebraic formulation of distraction lead one to think that it detects Cohen-Macaulayness? It ought to because regular sequences connect a single object, the polarization, to both $S / I$ and $S / \tilde{I}$. This connection has been observed before; it was noted explicitly in the Introduction to [BCR05], for instance.

Definition 5.6. For a variable $t$ and $a \in \mathbb{N}$, the polarization of the pure power $t^{a}$ is $t_{a}^{\prime}=t_{0} t_{1} t_{2} \cdots t_{a-1}$, a squarefree monomial in $a$ new variables $t^{\prime}=t_{0}, \ldots, t_{a-1}$. For 
$\mathbf{a} \in \mathbb{N}^{n}$, the polarization of the monomial $\mathbf{x}^{\mathbf{a}} \in S$ is the product

$$
\mathbf{x}_{\mathbf{a}}^{\prime}=\prod_{i=1}^{n}\left(x_{i}^{\prime}\right)_{a_{i}} \in S^{\prime}
$$

of the polarizations of its pure-power factors $x_{i}^{a_{i}}$, where $S^{\prime}$ is a polynomial ring in variables $x_{i j}$ for $i=1, \ldots, n$ and $j=0, \ldots, a_{i}-1$. The polarization $I^{\prime} \subseteq S^{\prime}$ of a monomial ideal $I \subseteq S$ is generated by the polarizations of its minimal generators.

EXAMPLE 5.7. The polarization of $I=\left\langle x^{3} y z^{2}, y^{2} z^{4}\right\rangle$ is

$$
I^{\prime}=\left\langle x_{0} x_{1} x_{2} y_{0} z_{0} z_{1}, y_{0} y_{1} z_{0} z_{1} z_{2} z_{3}\right\rangle .
$$

It is well-known that the quotient by a monomial ideal $I$ is Cohen-Macaulay if and only if the quotient by its polarization $I^{\prime}$ is; see [MiSt05, Exercise 3.15] or [Kum08, Proposition 1.3.4]. The idea of the proof is that the elements $x_{i j}-x_{i 0}$ form a regular sequence in $S^{\prime} / I^{\prime}$, and the quotient modulo this regular sequence is $S / I$, if one sets $x_{i 0}=x_{i}$ for all $i$. This argument in fact shows that a minimal free resolution of $S^{\prime} / I^{\prime}$ over $S^{\prime}$ descends to a minimal free resolution of $S / I$ over $S$, so most of the homological invariants - Betti numbers, and so on - descend, as well. A similar procedure relates the polarization to the distraction, so Corollary 5.5 is not surprising from this perspective, although it is harder than one might imagine to prove it directly via regular sequences. Indeed, $S / \tilde{I}$ is not a local ring, so the implication " $V_{I}$ is Cohen-Macaulay $\Rightarrow S / I^{\prime}$ is Cohen-Macaulay" isn't obvious.

The geometric characterization of the Cohen-Macaulay condition via distracting arrangements in Corollary 5.1 gives rise to another geometric characterization, also observed by Berkesch and Matusevich [BeMa08, Corollary 2.15]: when $V_{I}$ is sliced by all translates of a sufficiently general linear subspace of dimension $n-\operatorname{dim}(S / I)$, the number of points in the intersection - counted with multiplicityis constant precisely when $S / I$ is Cohen-Macaulay. The following generalizes their result, which had additional hypotheses (such as integrality) on the projection $A$.

Proposition 5.8. Let $\mathbb{k}=\mathbb{C}$. If a linear map $A: \mathbb{C}^{n} \rightarrow \mathbb{C}^{d}$ takes every coordinate subspace in the zero set $Z(I)$ isomorphically to $\mathbb{C}^{d}$, then every fiber of the projection $V_{I} \rightarrow \mathbb{C}^{d}$ has Krull dimension $0 . S / I$ is Cohen-Macaulay of dimension d exactly when every fiber has multiplicity $\operatorname{deg}(I)$ equal to the standard $\mathbb{Z}$-graded degree of $I$.

Proof. $S / I$ is Cohen-Macaulay if and only if $V_{I}$ is, by Corollary 5.1 along with Corollary 5.5 and its proof. The projection $A$ makes $V_{I}$ into a family over the smooth base $\mathbb{C}^{d}$. Since $V_{I}$ is a finite union of subspaces parallel to coordinate spaces in $Z(I)$, this family is finite over $\mathbb{C}^{d}$. Under these conditions, the CohenMacaulay condition on $V_{I}$ is equivalent to the flatness of this family (see [BrHe93, Proposition 2.2.11] or [MiSt05, Theorem 13.37.5]), which in turn is equivalent to the constancy of the multiplicity of the fibers [Har77, Proposition III.9.2(e) and Exercise II.5.8]. The constant multiplicity must be $\operatorname{deg}(I)$ because this is the number of dimension $d$ irreducible components of $V_{I}$.

The statement from which Berkesch and Matusevich derive most of their consequences, such as the second half of Corollary 5.1 and their version of Proposition 5.8, is a coarsening [BeMa08, Corollary 2.14] of Theorem 4.7 to a $\mathbb{Z}^{d}$-graded setting. The situation is that of Proposition 5.8, except that $A$ is required to be represented by a $d \times n$ integer matrix, also called $A$, whose columns span $\mathbb{Z}^{d}$ and whose kernel contains no nonzero positive vectors. Such a matrix $A$ induces a $\mathbb{Z}^{d}$-grading on $S$, 
in which each monomial $\mathbf{x}^{\mathbf{a}}$ has degree $A \cdot \mathbf{a} \in \mathbb{Z}^{d}$. Any module $N$ that is $\mathbb{Z}^{d}$-graded has true degrees $\operatorname{tdeg}_{d}(N)=\left\{\mathbf{u} \in \mathbb{Z}^{d} \mid N_{\mathbf{u}} \neq 0\right\}$ and quasidegrees $\operatorname{qdeg}_{d}(N)=$ Zariski closure of $\operatorname{tdeg}_{d}(N)$ in $\mathbb{C}^{d}$.

Lemma 5.9. Fix a $\mathbb{Z}^{n}$-graded finitely generated $S$-module $M$ of dimension at most $d$, or an artinian $\mathbb{Z}^{n}$-graded module whose $\mathbb{Z}^{n}$-graded Matlis dual has dimension at most $d$. Then $\operatorname{qdeg}_{d}(M)=A(\operatorname{qdeg}(M))$ is the image in $\mathbb{C}^{d}$ of the $\mathbb{C}^{n}$-quasidegrees.

Proof. By Matlis duality, assume $M$ is finitely generated. The set $\operatorname{tdeg}_{n}(M)$ of true degrees of $M$ admits a decomposition as a finite union of positive integer orthants having the form $\alpha+\mathbb{N}^{F}$, where $\alpha \in \mathbb{Z}^{n}$ and $|F| \leq d$. Any such decomposition yields a decomposition of $\operatorname{tdeg}_{d}(M)$ as a finite union of translated semigroups $\beta+\mathbb{N} A_{F}$, where $A_{F}$ is the set of columns of $A$ indexed by $F$. The result holds because Zariski closure commutes with the application of $A$ to such sets.

Here, finally, is a precise formulation of [BeMa08, Corollary 2.14].

Corollary 5.10. Fix a $\mathbb{Z}^{d}$-grading by $A$. In the situation of Proposition 5.8, the fiber over $\gamma \in \mathbb{C}^{d}$ has multiplicity $>\operatorname{deg}(I)$ exactly when $\gamma \in \bigcup_{i=0}^{d-1} \operatorname{qdeg}_{d}\left(H_{\mathfrak{m}}^{i}(S / I)\right)$.

Proof. The multiplicity of the fiber $V_{I}(\gamma)$ over $\gamma \in \mathbb{C}^{d}$ is $>\operatorname{deg}(I)$ if and only if $V_{I}$ fails to be flat near $\gamma$ as a family over $\mathbb{C}^{d}$. This occurs precisely when the stalk of the coordinate ring $\mathbb{C}\left[V_{I}\right]=S / \tilde{I}$ near $\gamma$ fails to be Cohen-Macaulay over the local ring of $\gamma \in \mathbb{C}^{d}$. This condition is equivalent to the failure of the Cohen-Macaulay condition for $V_{I}$ locally at some point $\alpha \in \mathbb{C}^{n}$ mapping to $\gamma$ under $A$. But we have already seen, in Theorem 4.7, that the set of points in $V_{I}$ where the CohenMacaulay condition fails is the union, over $i=0, \ldots, d-1$, of the quasidegree sets $\operatorname{qdeg}\left(H_{\mathfrak{m}}^{i}(S / I)\right)$ in $\mathbb{C}^{n}$. The result now follows from Lemma 5.9.

\section{Dual Čech simplicial complexes}

What would a survey about topological criteria for Cohen-Macaulayness be without some mention of Alexander duality? Well, here it is, in a third family of simplicial complexes that has appeared in the literature and whose cohomology detects the Cohen-Macaulay property for an arbitrary monomial ideal $I$.

Definition 6.1. Write $\bar{\Lambda}_{I}=\mathbb{N}^{n} \backslash \Lambda_{I}$ for the set of exponent vectors on the monomials in $I$. Given $\mathbf{b} \in \mathbb{Z}^{n}$, write $\mathbf{b}=\mathbf{b}_{+}-\mathbf{b}_{-}$as a difference of vectors in $\mathbb{N}^{n}$. The dual Čech simplicial complex for $\mathbf{a} \in \mathbb{Z}^{n}$ is

$$
\check{\mathrm{C}} \Delta^{\mathbf{a}}=\left\{F \subseteq\{1, \ldots, n\} \mid F=\operatorname{supp}\left(\mathbf{b}_{-}\right) \text {for some } \mathbf{b} \in \mathbf{a}+\bar{\Lambda}_{I}\right\} .
$$

As suggested by the nomenclature, Definition 6.1 is connected to Cech simplicial complexes by Alexander duality, as we shall see later in this section.

Definition 6.2. The Alexander dual $\Delta^{*}$ of a simplicial complex $\Delta$ is characterized by the following property: $F \notin \Delta$ if and only if $\bar{F} \in \Delta^{*}$.

Alexander duality for simplicial complexes entered into the earliest of Hochster's investigations into monomial commutative algebra [Hoc77], sometimes implicitly. The late 1990s saw a resurgence of explicit manifestations of Alexander duality, spurred in large part by the Eagon-Reiner theorem [ER98]: possessing a linear free resolution is dual to being Cohen-Macaulay. In what follows, only the most pertinent aspects of Alexander duality are mentioned; for additional background - both mathematical and historical - see [MiSt05, Chapter 5], including the Notes there. 
The family of simplicial complexes in Definition 6.1 was identified by Mustaţă [Mus00, Section 2] for the purpose of a fundamental calculation, made independently by Terai [Ter99], of the local cohomology of the polynomial ring $S$ with support on a (squarefree) monomial ideal. The resulting Hilbert series formula greatly resembles Hochster's formula for the local cohomology of a Stanley-Reisner ring (Theorem 3.1). The relation between the Mustaţă-Terai formula and Hochster's formula is a functorial effect of Alexander duality on local cohomology, known as local duality with monomial support [Mil00, Section 6]. This duality, in turn, is a combinatorial special case of Greenlees-May duality [GM92, Mil02a], which is generally an ajointness between the derived functors of completion at an ideal $I$ and of taking support on $I$.

Theorem 6.3. If $\mathbf{a} \in \mathbb{Z}^{n}$ and $\mathbb{1}=(1, \ldots, 1)$, then the dual Čech simplicial complex $\check{C} \Delta^{-\mathbf{a}-\mathbb{1}}$ is Alexander dual to $\check{C} \Delta_{\mathbf{a}}$ inside of the simplex on $\{1, \ldots, n\} \backslash \operatorname{supp}\left(\mathbf{a}_{-}\right)$.

Proof. $\bar{F} \in \check{\mathrm{C}} \Delta^{-\mathbf{a}-\mathbb{1}} \Leftrightarrow \operatorname{supp}\left(\mathbf{b}_{-}\right)=\bar{F}$ for some $\mathbf{b} \in-\mathbf{a}-\mathbb{1}+\bar{\Lambda}_{I}$

$$
\begin{aligned}
& \Leftrightarrow \mathbf{b}_{-}=-\bar{F} \text { for some } \mathbf{b} \in-\mathbf{a}-\mathbb{1}+\bar{\Lambda}_{I} \\
& \Leftrightarrow-\bar{F} \in \mathbf{b}+\mathbb{Z}^{F} \text { for some } \mathbf{b} \in-\mathbf{a}-\mathbb{1}+\bar{\Lambda}_{I} \\
& \Leftrightarrow-\bar{F} \in-\mathbf{a}-\mathbb{1}+\bar{\Lambda}_{I}+\mathbb{Z}^{F} \\
& \Leftrightarrow F-\mathbb{1} \in-\mathbf{a}-\mathbb{1}+\bar{\Lambda}_{I}+\mathbb{Z}^{F} \\
& \Leftrightarrow \mathbf{a}+F \in \bar{\Lambda}_{I}+\mathbb{Z}^{F} \\
& \Leftrightarrow \mathbf{a} \in \bar{\Lambda}_{I}+\mathbb{Z}^{F} \\
& \Leftrightarrow\left(I S_{F}\right)_{\mathbf{a}}=\mathbb{k} \\
& \Leftrightarrow\left(S_{F} / I S_{F}\right)_{\mathbf{a}}=0 . \\
& \Leftrightarrow F-\operatorname{supp}\left(\mathbf{a}_{-}\right) \notin \check{\mathrm{C}} \Delta_{\mathbf{a}} .
\end{aligned}
$$

All of the equivalences are elementary; the only one demanding verification of auxiliary data is the final line, for which it is important to check that $F$ actually contains $\operatorname{supp}\left(\mathbf{a}_{-}\right)$. This is not so bad, though: $\bar{F} \in \check{\mathrm{C}} \Delta^{-\mathbf{a}-\mathbb{1}}$ implies that $\bar{F} \subseteq$ $\operatorname{supp}\left((-\mathbf{a}-\mathbb{1})_{-}\right)=\operatorname{supp}\left((\mathbf{a}+\mathbb{1})_{+}\right)$, and this is the complement of $\operatorname{supp}\left(\mathbf{a}_{-}\right)$.

REMARK 6.4. If Mustaţă was trying to compute local cohomology with support on a squarefree monomial ideal, how did he come upon a formula for something equivalent to local cohomology — with maximal support - of the quotient by an arbitrary monomial ideal? Fairly easily: in computing the limit that defines the local cohomology $H_{I_{\Delta}}^{i}(S)$ supported on a squarefree monomial ideal $I_{\Delta}$, Mustaţă was led to compute a simplicial formula for $\operatorname{Ext}_{S}^{i}(S / I, S)$, where the monomial ideal $I$ is a Frobenius power of $I_{\Delta}$. But he also remarked that a similar formula holds for all monomial ideals $I$ [Mus00, Section 2]. The Alexander duality observed in this section is a direct result of $\mathbb{Z}^{n}$-graded local duality (the ordinary kind, as opposed to with monomial support):

$$
\begin{aligned}
\operatorname{Ext}^{i}(S / I, S)_{-\mathbf{a}-\mathbb{1}} & =\operatorname{Ext}^{i}(S / I, S(-\mathbb{1}))(\mathbb{1})_{-\mathbf{a}-\mathbb{1}} \\
& =\operatorname{Ext}^{i}(S / I, S(-\mathbb{1}))_{-\mathbf{a}} \\
& =\left(H_{\mathfrak{m}}^{n-i}(S / I)_{\mathbf{a}}\right)^{\vee} .
\end{aligned}
$$

The formula for $\operatorname{Ext}^{i}(S / I, S)$ is perhaps the simplest formula in this survey. 
Corollary 6.5. If $\mathbf{a} \in \mathbb{Z}^{n}$, then $\operatorname{Ext}^{i}(S / I, S)_{\mathbf{a}}=\widetilde{H}^{i-2}\left(\check{C} \Delta^{\mathbf{a}} ; \mathbb{k}\right)$.

Proof. The Alexander duality isomorphism (see [MiSt05, Theorem 5.6], for example) states that, for two simplicial complexes $\Delta$ and $\Delta^{*}$ that are Alexander dual inside of a simplex $\sigma$, there is a canonical isomorphism $\widetilde{H}_{i-1}(\Delta ; \mathbb{k})=\widetilde{H}^{|\sigma|-i-2}\left(\Delta^{*} ; \mathbb{k}\right)$. Now apply the Alexander duality in Theorem 6.3 to the simplicial formula in Theorem 3.8, using the local duality in Remark 6.4:

$$
\begin{aligned}
\operatorname{Ext}^{n-i}(S / I, S)_{-\mathbf{a}-\mathbb{1}} & =\left(H_{\mathfrak{m}}^{i}(S / I)_{\mathbf{a}}\right)^{\vee} \\
& =\widetilde{H}_{i-\left|\operatorname{supp}\left(\mathbf{a}_{-}\right)\right|-1}\left(\check{\mathrm{C}} \Delta_{\mathbf{a}} ; \mathbb{k}\right) \\
& =\widetilde{H}^{n-i-2}\left(\check{\mathrm{C}} \Delta^{-\mathbf{a}-1} ; \mathbb{k}\right) .
\end{aligned}
$$

Now, in the top and bottom lines, replace $n-i$ by $i$ and $\mathbf{a}$ by $-\mathbf{a}-\mathbb{1}$. (Avoid trying to do this in the middle line, which has a confusing $\operatorname{supp}\left(\mathbf{a}_{-}\right)$to deal with.)

REMARK 6.6. The formula in Corollary 6.5 seems to have been the first simplicial local cohomology - or equivalently, Ext - formula for arbitrary monomial ideals to appear in the literature [Mus00, Section 2], being a number of years earlier than the next, which seems to have been [Tak05, Theorem 1].

\section{Koszul simplicial complexes}

As we have seen, verifying the Cohen-Macaulay condition by analyzing the homology of a family of simplicial complexes, instead of a single simplicial complex as in the squarefree case, can be accomplished using a variety of simplicial complexes. The simplest family, however, is probably the following [MiSt05, Definition 5.9].

Definition 7.1. Given a vector $\mathbf{b} \in \mathbb{N}^{n}$, set $\mathbf{b}^{\prime}=\mathbf{b}-\operatorname{supp}(\mathbf{b})$. For any monomial ideal $I$, the (lower) Koszul simplicial complex of $S / I$ in degree $\mathbf{b} \in \mathbb{N}^{n}$ is

$$
\mathrm{K} \Delta_{\mathbf{b}}=\left\{\text { squarefree vectors } F \preceq \mathbf{b} \mid \mathbf{x}^{\mathbf{b}^{\prime}+F} \notin I\right\} .
$$

Equivalently, if $\Lambda_{I} \subseteq \mathbb{N}^{n}$ corresponds to the standard monomials (Definition 2.1),

$$
\mathrm{K} \Delta_{\mathbf{b}}=\left\{F \preceq \operatorname{supp}(\mathbf{b}) \mid \mathbf{b}^{\prime}+F \in \Lambda_{I}\right\} .
$$

The motivation for the definition is analogous to the Cech simplicial complexes: just as the algebraic chain complex of $\check{\mathrm{C}} \Delta_{\mathrm{a}}$ is the graded piece of the Čech complex of $S / I$ in degree $\mathbf{a}$, the algebraic cochain complex of $\mathrm{K} \Delta_{\mathbf{b}}$ is the graded piece of the Koszul complex of $S / I$ in degree $\mathbf{b}$. In fact, the analogy between these families of simplicial complexes is even closer.

Lemma 7.2. For $\mathbf{b} \in \mathbb{N}^{n}$ and $\mathbf{a}=\mathbf{b}-\mathbb{1}$, the Koszul simplicial complex at $\mathbf{b}$ equals

$$
\mathrm{K} \Delta_{\mathbf{b}}=\left\{F-\operatorname{supp}\left(\mathbf{a}_{-}\right) \mid(S / I)_{\mathbf{a}+F}=\mathbb{k}\right\} .
$$

Consequently, $\check{C} \Delta_{\mathbf{a}}$ is a subcomplex of $\mathrm{K} \Delta_{\mathbf{b}}$.

Proof. The displayed formula for $\mathrm{K} \Delta_{\mathbf{b}}$ follows immediately from Definition 7.1, given that $(S / I)_{\mathbf{a}+F}$ can only be nonzero if $\mathbf{a}+F$ has nonnegative coordinates, which occurs precisely when $F$ contains $\operatorname{supp}\left(\mathbf{a}_{-}\right)$. The containment $\check{\mathrm{C}} \Delta_{\mathbf{a}} \subseteq \mathrm{K} \Delta_{\mathbf{b}}$ follows from Definition 3.6, given that $\left(S_{F} / I S_{F}\right)_{\mathbf{a}}$ is nonzero precisely when $(S / I)_{\mathbf{a}+r F}$ is nonzero for all $r \geq 1$. 
In certain special cases, as we shall see in Corollary 7.8, below, the inclusion $\check{\mathrm{C}} \Delta_{\mathbf{a}} \subseteq \mathrm{K} \Delta_{\mathbf{a}+\mathbb{1}}$ induces an isomorphism on a particular cohomology group, and this cohomology is precisely what is needed to detect the Cohen-Macaulay condition, or its failure. In general, a graded $S$-module $M$ is Cohen-Macaulay precisely when its minimal free resolution of $M$ has length equal to the codimension of $M$ [MiSt05, Theorem 13.37.2]. Equivalently, the Betti numbers $\beta_{i, \mathbf{a}}(M)$ must vanish in homological degrees $i$ greater than the codimension of $M$, for all a. Homologically, the Betti number in degree $\mathbf{a}$ and homological degree $i$ is the $\mathbb{k}$-vector space dimension

$$
\beta_{i, \mathbf{a}}(M)=\operatorname{dim}_{\mathbb{k}} \operatorname{Tor}_{S}^{i}(\mathbb{k}, M)_{\mathbf{a}}
$$

[MiSt05, Lemma 1.32] (see also [MiSt05, Definition 8.22]). Therefore, the following generalization of Hochster's formula for $\operatorname{Tor}_{S}^{i}\left(\mathbb{k}, S / I_{\Delta}\right)_{\mathbf{a}}$ to arbitrary monomial quotients $S / I$ [MiSt05, Theorem 5.11] yields the desired simplicial characterization of the Cohen-Macaulay condition in Corollary 7.4.

TheOREM 7.3. Given $\mathbf{b} \in \mathbb{N}^{n}$ with support $F=\operatorname{supp}(\mathbf{b})$, a simplicial formula for the Betti number of $S / I$ in degree $\mathbf{b}$ is given by the $\mathbb{k}$-vector space dimension of

$$
\operatorname{Tor}_{i}(S / I, \mathbb{k})_{\mathbf{b}}=\widetilde{H}^{|F|-i-1}\left(\mathrm{~K} \Delta_{\mathbf{b}} ; \mathbb{k}\right) .
$$

Proof. As mentioned after Definition 7.1, this can be proved by direct Koszul complex calculation. (The proof of [MiSt05, Theorem 5.11] actually proceeds via Alexander duality; see Remark 7.10.)

COROLlary 7.4. $S / I$ is Cohen-Macaulay if and only if $\widetilde{H}^{|F|-i-1}\left(\mathrm{~K} \Delta_{\mathbf{b}} ; \mathbb{k}\right)=0$ for all $\mathbf{b} \in \mathbb{N}^{n}$ and all $i>n-d$, where $d=\operatorname{dim}(S / I)$ and $F=\operatorname{supp}(\mathbf{b})$.

For the record, Hochster's Tor formula is the special case of Theorem 7.3 in which $I=I_{\Delta}$ is squarefree.

Corollary 7.5 (Hochster's Tor formula). The nonzero Betti numbers of $S / I_{\Delta}$ lie only in squarefree degrees $F$, and we have

$$
\operatorname{Tor}_{i}\left(S / I_{\Delta}, \mathbb{k}\right)_{F}=\widetilde{H}^{|F|-i-1}\left(\left.\Delta\right|_{F} ; \mathbb{k}\right),
$$

where $\Delta_{F}$ is the subcomplex of all faces of $\Delta$ whose vertices lie in $F$.

PROOF. This was originally proved in [Hoc77]. It follows immediately from Theorem 7.3 and Definition 7.1. For additional information and context, this result is also [Sta96, Theorem II.4.8], [BrHe93, Remark 5.5.5], and [MiSt05, Corollary 5.12].

REMARK 7.6. For general vectors $\mathbf{b} \in \mathbb{N}^{n}$ and $\mathbf{a}=\mathbf{b}-\mathbb{1}$, the relationship between the simplicial complexes $\check{\mathrm{C}} \Delta_{\mathbf{a}}$ and $\mathrm{K} \Delta_{\mathbf{b}}$ in Lemma 7.2 is mysterious, and perhaps difficult to analyze (if such an analysis is possible, it would be interesting to see). This is to be expected, since the Čech simplicial complexes compute local cohomology (Theorem 3.8), whereas the Koszul simplicial complexes compute Tor into $\mathbb{k}$ (Theorem 7.3), which behaves quite differently from local cohomology, as a function of the $\mathbb{Z}^{n}$-graded degree.

Remark 7.6 notwithstanding, the socle of the first nonzero local cohomology precisely reflects the top Betti numbers. This holds quite generally for arbitrary finitely generated modules over regular local or graded rings. Here is the $\mathbb{Z}^{n}$-graded version. Its proof demonstrates that this result is a consequence of duality for resolutions [Mil00, Theorem 4.5] of $\mathbb{Z}^{n}$-graded modules. That result was developed in the context of Alexander duality for arbitrary (that is, not necessarily squarefree) 
monomial ideals and modules, in response to the Eagon-Reiner theorem [ER98] and its generalizations. The point is that a minimal $\mathbb{Z}^{n}$-graded injective resolution of a finitely generated module $M$ contains data equivalent to a minimal injective resolution of its Alexander dual, and therefore also enough data to recover minimal free resolutions of both $M$ and its Alexander dual. These considerations include the fact - key in the proof of Theorem 7.7 - that a minimal free resolution of $M$ is equivalent to the maximal-support part of a minimal injective resolution of $M$.

THEOREM 7.7. Let $M$ be finitely generated and $\mathbb{Z}^{n}$-graded of projective dimension $p$ $\left(\beta_{p, \mathbf{b}}(M) \neq 0\right.$ for some $\mathbf{b} \in \mathbb{Z}^{n}$, and $\beta_{i, \mathbf{b}}(M)=0$ for $\left.i>p\right)$. There is a natural map

$$
\operatorname{Tor}_{i}(M, \mathbb{k})_{\mathbf{b}} \rightarrow H_{\mathfrak{m}}^{n-i}(M)_{\mathbf{b}-\mathbb{1}}
$$

for all $i$ and all $\mathbf{b} \in \mathbb{Z}^{n}$. If $i=p$, and $\mathbf{b}$ is any degree such that the Betti number $\beta_{p, \mathbf{b}}(M)$ is nonzero, then the natural map is an isomorphism:

$$
\operatorname{Tor}_{p}(M, \mathbb{k})_{\mathbf{b}} \cong H_{\mathfrak{m}}^{n-p}(M)_{\mathbf{b}-\mathbb{1}} \text { if } \beta_{p, \mathbf{b}} \neq 0 .
$$

Proof. Let $F$. be a minimal free resolution of $M$. Then $\operatorname{Tor}_{i}(M, \mathbb{k})$ is the homology $H_{i}(F \bullet \otimes \mathbb{k})$. In constrast, by [Mil00, Theorem 4.5.5], the $\mathbb{Z}^{n}$-graded translate $H_{\mathfrak{m}}^{n-i}(M)(-\mathbb{1})$ of the local cohomology up by $\mathbb{1}$ is the homology $H_{i}\left(F_{\bullet} \otimes E_{\mathbb{k}}\right)$, where $E_{\mathbb{k}}$ is the $\mathbb{Z}^{n}$-graded injective hull of $\mathbb{k}$, also known as the Matlis dual of $S$. The existence of the natural map follows simply from the canonical inclusion of $\mathbb{k}$ as the socle of $E_{\mathbb{k}}$. The natural map is an isomorphism when $i=p$ is maximal because, by minimality of the resolution, the differentials of $F_{\bullet} \otimes E_{\mathrm{k}}$ are zero on the socle.

As a consequence, we find that the mysterious inclusion from Lemma 7.2 has at least one tractable feature. Conveniently, this feature pertains precisely to the cohomology that reflects the Cohen-Macaulay property for $S / I$ (Corollary 7.4).

Corollary 7.8. Let $S / I$ have projective dimension $p$. Assume that $\beta_{p, \mathbf{b}}(S / I) \neq 0$. Writing $\mathbf{a}=\mathbf{b}-\mathbb{1}$ and $F=\operatorname{supp}(\mathbf{b})$, the inclusion $\check{C} \Delta_{\mathbf{a}} \subseteq \mathrm{K} \Delta_{\mathbf{b}}$ induces an isomorphism $\widetilde{H}^{|F|-p-1}\left(\check{C} \Delta_{\mathbf{a}} ; \mathbb{k}\right)=\widetilde{H}^{|F|-p-1}\left(\mathrm{~K} \Delta_{\mathbf{b}} ; \mathbb{k}\right)$ on cohomology.

REMARK 7.9. It is plausible that the Cech and lower Koszul simplicial complexes in Corollary 7.8 could be equal, but neither a proof nor a counterexample is known. It is also possible that additional hypotheses on $S / I$ might be required; starting with the case where $S / I$ is Cohen-Macaulay is probably a good idea.

REMARK 7.10. The formula in Theorem 7.3 comes in two flavors [MiSt05, Theorems 1.34 and 5.11] that are dual by definition, the latter derived as an immediate consequence of the former, by Alexander duality. Hence it would be possible - but more or less trivial - to present dual versions of the results in this section. In contrast, the space devoted to the Čech simplicial complexes $\check{\mathrm{C}} \Delta_{\mathbf{a}}$ and their duals $\check{\mathrm{C}} \Delta^{\mathbf{a}}$ in earlier sections was worthwhile: they arose separately, and their duality is neither by definition nor self-evident, being a manifestation of local duality (Remark 6.4).

\section{References}

[Ado94] Alan Adolphson, Hypergeometric functions and rings generated by monomials, Duke Math. J. 73 (1994), no. 2, 269-290.

[BaWe02] Ekkehard Batzies and Volkmar Welker, Discrete Morse theory for cellular resolutions, J. Reine Angew. Math. 543 (2002), 147-168.

[BPS98] Dave Bayer, Irena Peeva, and Bernd Sturmfels, Monomial resolutions, Math. Res. Lett. 5 (1998), no. 1-2, 31-46. 
[BaSt98] Dave Bayer and Bernd Sturmfels, Cellular resolutions of monomial modules, J. Reine Angew. Math. 502 (1998), 123-140.

[BeMa08] Christine Berkesch and Laura Matusevich, A-graded methods for monomial ideals, preprint, 2009. arXiv:math.AC/0807.4306v2

[BCR05] Anna Bigatti, Aldo Conca, and Lorenzo Robbiano, Generic initial ideals and distractions, Comm. Algebra 33 (2005), no. 6, 1709-1732.

[BrHe93] Winfried Bruns and Jürgen Herzog, Cohen-Macaulay rings, Cambridge Studies in Advanced Mathematics Vol. 39, Cambridge University Press, Cambridge, 1993.

[DMM08] Alicia Dickenstein, Laura Felicia Matusevich, and Ezra Miller, Binomial D-modules, preprint. arXiv:math.AG/0610353

[ER98] John A. Eagon and Victor Reiner, Resolutions of Stanley-Reisner rings and Alexander duality, J. Pure Appl. Algebra 130 (1998), no. 3, 265-275.

[Flø06] Gunnar Fløystad, Cohen-Macaulay cell complexes, Algebraic and geometric combinatorics, 205-220, Contemp. Math., 423, Amer. Math. Soc., Providence, RI, 2006.

[GGZ87] I. M. Gelfand, M. I. Graev, and A. V. Zelevinsky, Holonomic systems of equations and series of hypergeometric type, Dokl. Akad. Nauk SSSR 295 (1987), no. 1, 14-19.

[GKZ89] I. M. Gelfand, A. V. Zelevinsky, and M. M. Kapranov, Hypergeometric functions and toric varieties, Funktsional. Anal. i Prilozhen. 23 (1989), no. 2, 12-26. Correction in ibid, 27 (1993), no. 4, 91.

[Grä84] Hans-Gert Gräbe, The canonical module of a Stanley-Reisner ring, J. Algebra 86 (1984), 272-281.

[GM92] John P. C. Greenlees and J. Peter May, Derived functors of I-adic completion and local homology, J. Algebra 149 (1992), no. 2, 438-453.

[Har77] Robin Hartshorne, Algebraic geometry, Graduate Texts in Mathematics Vol. 52, Springer-Verlag, New York, 1977.

[Hoc77] Melvin Hochster, Cohen-Macaulay rings, combinatorics, and simplicial complexes, Ring theory, II (Proc. Second Conf., Univ. Oklahoma, Norman, Okla., 1975) (B. R. McDonald and R. Morris, eds.), Lecture Notes in Pure and Applied Mathematics Vol. 26, Marcel Dekker, New York, 1977, pp. 171-223.

[Ish80] Masa-Nori Ishida, Torus embeddings and dualizing complexes, Tôhoku Math. J. (2) 32 (1980), no. 1, 111-146.

[Ish87] Masa-Nori Ishida, The local cohomology groups of an affine semigroup ring, Algebraic geometry and commutative algebra in Honor of Masayaoshi Nagata, Vol. I, Kinokuniya, Tokyo, 1987, pp. 141-153.

[JoMi08] Shin-Yao Jow and Ezra Miller, Multiplier ideals of sums via cellular resolutions, Math. Res. Lett. 15 (2008), no. 2, 359-373.

[Kos50] Jean-Louis Koszul, Homologie et cohomologie des algèbres de Lie, Bull. Soc. Math. France 78 (1950), 65-127.

[Kum08] Manoj Kummini, Homological invariants of monomial and binomial ideals, PhD thesis, University of Kansas, 2008.

[MMW05] Laura Felicia Matusevich, Ezra Miller, and Uli Walther, Homological methods for hypergeometric families, J. Amer. Math. Soc. 18 (2005), no. 4, 919-941.

[Mil00] Ezra Miller, The Alexander duality functors and local duality with monomial support, J. Algebra 231 (2000), 180-234.

[Mil02a] Ezra Miller, Graded Greenlees-May duality and the Čech hull, Local cohomology and its applications (Guanajuato, 1999), Lecture Notes in Pure and Applied Mathematics Vol. 226, Marcel Dekker, New York, 2002, pp. 233-253.

[Mil02b] Ezra Miller, Cohen-Macaulay quotients of normal semigroup rings via irreducible resolutions, Math. Res. Lett. 9 (2002), no. 1, 117-128.

[MiSt05] Ezra Miller and Bernd Sturmfels, Combinatorial Commutative Algebra, Graduate Texts in Mathematics, vol. 227, Springer-Verlag, New York, 2005.

[MSY00] Ezra Miller, Bernd Sturmfels, and Kohji Yanagawa, Generic and cogeneric monomial ideals, J. Symbolic Comput. 29 (2000), 691- 708.

[Mus00] Mircea Mustaţă, Local cohomology at monomial ideals, J. Symbolic Comput. 29 (2000), 709-720.

[Rah07] Ahad Rahimi, Tameness of local cohomology of monomial ideals with respect to monomial prime ideals, J. Pure Appl. Algebra 211 (2007), no. 1, 83-93. 
[Rei76] Gerald Allen Reisner, Cohen-Macaulay quotients of polynomial rings, Adv. Math. 21 (1976), no. 1, 30-49.

[SST00] Mutsumi Saito, Bernd Sturmfels, and Nobuki Takayama, Gröbner deformations of hypergeometric differential equations, Algorithms and Computation in Mathematics, vol. 6, Springer-Verlag, Berlin, 2000.

[Sta82] Richard P. Stanley, Linear Diophantine equations and local cohomology, Invent. Math. 68 (1982), 175-193.

[Sta96] Richard P. Stanley, Combinatorics and commutative algebra, second ed., Progress in Mathematics Vol. 41, Birkhäuser, Boston, MA, 1996.

[STV95] Bernd Sturmfels, Ngô Viêt Trung, and Wolfgang Vogel, Bounds on degrees of projective schemes, Math. Ann. 302 (1995), no. 3, 417-432.

[Tak05] Yukihide Takayama, Combinatorial characterizations of generalized Cohen-Macaulay monomial ideals, Bull. Math. Soc. Sci. Math. Roumanie (N.S.) 48(96) (2005), no. 3, 327-344.

[Tay60] Diana Taylor, Ideals generated by monomials in an R-sequence, Ph.D. thesis, University of Chicago, 1960.

[Ter99] Naoki Terai, Local cohomology modules with respect to monomial ideals, preprint, 1999.

[HVZ08] Jürgen Herzog, Marius Vladoiu, and Xinxian Zheng, How to compute the Stanley depth of a monomial ideal, J. Algebra (2008), DOI: 10.1016/j.jalgebra.2008.01.006

[Yan00] Kohji Yanagawa, Alexander duality for Stanley-Reisner rings and squarefree $\mathbb{N}^{n}$-graded modules, J. Algebra 225 (2000), no. 2, 630-645.

School of Mathematics, University of Minnesota, Minneapolis, MN 55455

Current address: Department of Mathematics, Duke University, Durham, NC 27708

E-mail address: ezra@math.duke.edu 